Radial and Nonradial Pulsations as Probes of Stellar Physics

ASP Conference Series, Vol. 259, 2002

C. Aerts, T.R. Bedding, \& J. Christensen-Dalsgaard, eds.

\title{
Multi-Site Observations of the Delta Scuti Stars V624 Tauri and HD 23194
}

\author{
L. Fox Machado ${ }^{1}$, M. Álvarez ${ }^{2}$,E. Michel ${ }^{3}$, Z.P. $\mathrm{Li}^{4}$, F. Pérez \\ Hernández ${ }^{1,5}$, M. Chevreton ${ }^{6}$, J.A. Belmonte ${ }^{1}$, R. Alonso ${ }^{1}$, C. Barban ${ }^{3}$, \\ N. Dolez ${ }^{7}$, A. Fernandez ${ }^{6}$, J.P. Guo ${ }^{4}$, M. Haywood ${ }^{3}$, Y.Y. Liu ${ }^{4}$, S. Pau ${ }^{6}$, \\ H. Planas ${ }^{2}$, B. Servan ${ }^{3}$, J.-C. Suárez ${ }^{3}$ \\ ${ }^{1}$ Instituto de Astrofísica de Canarias, E-38200 La Laguna, Tenerife, \\ Spain: ${ }^{2}$ Instituto de Astronomía de la UNAM, Ap.P. 877, Ensenada, \\ $B C$, Mexico; ${ }^{3}$ Observatoire de París-Meudon, DASGAL, UMR 8633, \\ F-92195 Meudon, France; ${ }^{4}$ Beijing Observatory, Chinese Academy of \\ Sciences, Beijing, P.R. China; ${ }^{5}$ Departamento de Astrofisica, \\ Universidad de La Laguna, Tenerife, Spain; ${ }^{6}$ Observatoire de París \\ -Meudon, DAEC, UMR 8632, F-92195 Meudon, France; ${ }^{7}$ Observatoire \\ Midi-Pyrénées, F-31400 Toulouse, France
}

\begin{abstract}
Some results of the photometry multi-site observations of two $\delta$ Scuti stars,V624 Tau and HD 23194, are presented. The observations were carried out in the framework of a STEPHI network in 1999. We collected 343 hours of useful data and detected seven frequencies in V624 Tau and two frequencies in HD 23194.
\end{abstract}

\section{Introduction}

The main purpose of multi-site observations of $\delta$ Scuti stars is to obtain detailed information on frequency spectrum of this type of variables. To this aim, V624 Tau and HD 23194, two main sequence $\delta$ Scuti stars in the Pleiades cluster, were observed in STEPHI X multi-site campaign in 1999. HD 23246 was used as comparison star. V624 Tau and HD 23194 are short period pulsating variables with magnitudes $\mathrm{V}=8.22$ and 8.17 , and oscillation amplitudes $\Delta \mathrm{V}=10 \mathrm{mmag}$ and $5 \mathrm{mmag}$ respectively (Rodriguez et al., 2000). The $\delta$ Scuti pulsations in V624 Tau were reported by Breger (1972) during a study of variable stars in the Pleiades cluster. The variability of HD 23194 was found by Koen et al. (1999).

\section{Observations and frequency analysis}

The campaign spanned the period 1999, November 27-December 30. As was done in previous STEPHI campaigns, three observatories approximately equallyspaced in longitude were operating during observations. These are: San Pedro Mártir, in Baja California, Mexico, Xing Long Station in Beijing, China; and Teide Observatory in Tenerife, Spain. The observational procedure and the data reduction were the same as in the previous campaigns (see Álvarez et al., 1998 for details). A total amount of 343 hours of useful data were collected from three 
sites. The coverage was $41.3 \%$ of the cycle. The amplitude spectrum of the time series was obtained by using the iterative sine wave fit (ISWF). The spectral resolution reached in this campaign is $\Delta \nu=0.51 \mu \mathrm{Hz}$. The frequencies have been obtained by using the prewhitening process, as explained in Álvarez et al. (1998), where the frequency peaks above a $99 \%$ confidence level are selected and subtracted iteratively from the original series until the whole spectrum is below this level. Detected frequencies are given in Table 1.

\section{Conclusions}

V624 Tau and HD 23194 have been found to be multi-mode pulsators with seven frequencies and two frequencies above $99 \%$ confedence level respectively. Theoretical work using this observational material is currently being developed (Suárez et al., these proceedings).

Table 1. Modes detected in our target stars above a $99 \%$ confidence level. $\mathrm{A}$ is the amplitude, $\mathrm{S} / \mathrm{N}$ is signal-to-noise ratio.

\begin{tabular}{lcccc}
\hline Star & & $\begin{array}{c}\nu \\
(\mu \mathrm{Hz})\end{array}$ & $\begin{array}{c}\mathrm{A} \\
(\mathrm{mmag})\end{array}$ & $\mathrm{S} / \mathrm{N}$ \\
\hline V624 Tau & $\nu_{1}$ & 242.9 & 1.5 & 8.2 \\
& $\nu_{2}$ & 409.0 & 0.9 & 7.2 \\
& $\nu_{3}$ & 413.5 & 2.2 & 17.5 \\
& $\nu_{4}$ & 416.4 & 0.8 & 6.3 \\
& $\nu_{5}$ & 451.7 & 1.2 & 9.7 \\
& $\nu_{6}$ & 489.4 & 1.5 & 13.0 \\
& $\nu_{7}$ & 529.1 & 0.7 & 6.8 \\
\hline HD 23194 & $\nu_{1}$ & 533.6 & 2.0 & 9.4 \\
& $\nu_{2}$ & 574.9 & 1.5 & 6.7 \\
\hline
\end{tabular}

\section{References}

Álvarez, M., Hernández, M.M., Michel, E., et al. 1998, A\&A, 340, 149 Breger, M. 1972, ApJ, 176, 367

Koen, C., Van Rooyen, R., Van Wyk, F., et al. 1999, MNRAS, 309, 1051

Rodríguez, E., López-González, M.J., López de Coca, P., 2000, A\&AS, 144, 469 